\title{
Diagnóstico geológico minero ambiental de las canteras de caliza en el departamento del Cesar
}

Martha Lucía Mendoza Castro', Islem Issela Urbina Garrido²

\section{Resumen}

El presente diagnóstico fue elaborado con el fin de brindar un soporte a las canteras para mejorar y analizar las dificultades que estas han estado presentando en la parte geológica, minera y ambiental. Para lo anterior, es necesario conocer si en la minería a mediana escala se están haciendo los estudios geológicos pertinentes, además, comparar los métodos de explotación de la caliza y determinar los impactos ambientales generados; cabe resaltar que esta investigación es cuantitativa y se rige bajo la correlación bibliográfica y empleo de lista de chequeo. Ahora bien, las canteras no realizan estudios geoquímicos por el tipo de roca, sin embargo, realizan análisis químicos y de calidad para cemento, base de construcción, hormigón, y para procesos químicos de cal viva. Los métodos de explotación se clasifican en banco escalonado ascendente y banco escalonado descendente. Por último, a nivel ambiental se lograron determinar impactos de polución, desforestación y alteración paisajística. En conclusión, las canteras emplean los estudios geológicos de acuerdo con su necesidad, además de que el método de explotación más recomendado es el de banco escalonado descendente. Así mismo, se identificó, a partir de la correlación con la matriz de Leopold (1971), que en la etapa en la que se presenta más impacto es en la de construcción y montaje.

Palabras clave: ambiental, banco, caliza, descendente, diagnóstico, geología, impactos, minería.

${ }^{1}$ Administradora de empresas, magíster en Gerencia de Proyectos $1+D+1$, docente investigadora de la Fundación Universitaria del Área Andina. Valledupar, Colombia. Correo: mmendoza27Dareandina.edu.co

Estudiante de Ingeniería Geológica de la Fundación Universitaria del Área Andina. Valledupar, Colombia. Correo: iurbina2la estudiantes.areandina.edu.co 


\section{Introducción}

El departamento del Cesar posee un gran potencial geológico debido a la creación de montañas, proceso geológico conocido como orogénesis y que trae consigo variedad de preciados minerales encontrados en el subsuelo y que actualmente se observan en forma de afloramiento, dando razón así a las explotaciones para uso y aplicaciones de estos mismos; entre ellos podemos encontrar la roca caliza, la cual pertenece al gremio de las rocas sedimentarias orgánicas, con un alto contenido de carbonato de calcio, aproximadamente un $50 \%$, lo que genera su efervescencia al contacto con ácido clorhídrico $(\mathrm{HCl})$, material usado para la construcción; además, también sirve como decoración para las paredes y pisos, esto corresponde más cuando se habla del mármol como tal.

No obstante, el área del departamento se encuentra distribuida en cuatro Unidades asociadas a la Geología que las caracteriza: Sierra Nevada de Santa Marta, Valle de los Ríos Cesar y Magdalena, La Serranía del Perijá y la Región Norte de la Cordillera Oriental; cada una con sus propios rasgos distintivos $\mathrm{y}$ diferente concepción en cuanto a los recursos minerales yacientes se refiere. (Guerrero, 2001)

El presente diagnóstico geológico minero ambiental de las canteras de caliza en el departamento del Cesar fue elaborado con el fin de observar un contexto regional, que busca enfocarse a la extracción de la roca caliza. Para la preparación de este documento se inició con la recopilación proveniente de tesis, informes, trabajos y libros referentes a la indagación del objeto de estudio del proyecto incursionado. De igual forma, en la compilación se ha hecho partícipe a organismos relacionados con la minería, como son: la Agencia Nacional de Minería, Corpocesar, Ministerio de Minas, entre otros. Además,

esta información, sumada a la observación en las visitas técnicas a las áreas de explotación minera, permitieron identificar aspectos importantes, que se convirtieron en la base para la elaboración de un formato de campo que facilitara acceder a la información que aquí se consigna. (Corponariño, s. f.)

Dentro de esta investigación se tendrán en cuenta los diferentes aspectos en las canteras de caliza ubicadas en el departamento del Cesar (minería a pequeña escala), dentro de los cuales 
se realizará un diagnostico geológico minero ambiental, para así poder evaluar y analizar estas diversas problemáticas que se están generando hoy en día, además de conocer si manejan una extracción o explotación de manera artesanal. Esto socorrerá a todas las canteras para mejorar, evitar y mitigar las dificultades que estas han estado presentando en la parte minerogeológica y ambiental en el Cesar.

De esta manera, se procede a presentar, desde el punto de vista práctico, el cual motivará y presentará a las distintas canteras, aquellos inconvenientes que afectan de manera directa e indirectamente al departamento del Cesar y con ello a Colombia, esto por medio del diagnóstico que está representado en la investigación, logrando así que con base a las conclusiones se mejore la producción y calidad de vida del artesano, aplicando técnicas de explotación del mineral; de la misma manera se reflejaría con el aumento de la fabricación como también en la contribución de un desarrollo sostenible y sustentable en la región.

Además, desde el punto de vista teórico de la investigación, se busca analizar los aspectos inherentes a los pequeños mineros desde la perspectiva de los impactos geológicos, mineros y ambientales que deja rastro en la explotación minera del Cesar para generar conocimientos con base en los resultados obtenidos.

También presenta un gran aporte social, ya que, haciendo uso de buenas técnicas de producción, se puede guiar a un mejor manejo de los recursos humanos, minerales y ambientales, hacer operativa la transferencia del crecimiento individual de las canteras y al profesional que lo asesora entornos fuera de su contexto de explotación.

Así mismo, Corponariño afirma que

este diagnóstico da una idea de la situación actual ambiental minera de los seis distritos que conforman el Distrito minero de la Llanada y puede convertirse en una herramienta importante para la elaboración de proyectos orientados a mejorar el aprovechamiento de los recursos y la identificación de nuevas alternativas tecnológicas del desarrollo minero acorde con la sostenibilidad ambiental.

Desde el punto de vista metodológico, esta investigación utilizará técnicas e instrumentos que permitirán lograr los objetivos en el 
orden teórico y aplicativo, este tiene como objeto primordial proponer un sistema conceptual explicativo en torno al diagnóstico geológico minero ambiental de las canteras de caliza, ubicadas en el departamento del Cesar $\mathrm{y}$, de esta manera, poder convertir las debilidades identificadas en fortalezas, aportando ideas a otros investigadores que se interesen por la recolección de datos y profundización de estos temas.

\section{Materiales y métodos}

En la actual investigación se aplica un estudio cuantitativo de tipo descriptivo, en el que Sampieri, Fernández y Baptista (2006), afirman que

la investigación descriptiva se emplea cuando el objetivo es el de detallar cómo son y cómo se manifiesta fenómenos, situaciones, contextos y eventos. Busca especificar propiedades, características y rasgos importantes de cualquier fenómeno que se analice. Se selecciona una serie de cuestiones y se mide o recolecta información sobre cada una de ellas, para describir lo que se investiga. Describe tendencias de un grupo o población.
Es oportuno ahora recalcar que la investigación se dirige a diagnosticar los aspectos geológicos, mineros y ambientales de la cantera de caliza en el departamento del Cesar, en el cual la indagación descriptiva accede a examinar las condiciones de las variables, mostrándose tal como se exhiben en la realidad sin elaborar deducciones correspondientes debido a que busca medir las variables, dimensiones e indicadores acerca de la extracción de la caliza, de tal manera que se valoren las medidas, los procesos, los estudios y demás factores inmersos a las constataciones antes expuestas para así generar predicciones especificas en forma de conclusiones, además de recomendaciones. No obstante, se integra con un estudio de correlación al procurar analizar los métodos de explotación en el que se busca interpretar cuál o cuáles son los más viables al momento de la extracción de la caliza.

Población. A partir de los títulos mineros registrados en el departamento del Cesar, se estimó que existen 35 canteras de caliza, las cuales se desglosan, según los contratos de legalización y concesión, así: 17 en etapa de exploración, cinco en etapa de construcción y montaje $\mathrm{y}$, por último, trece en etapa de explotación. 
Muestra. Para cumplir los objetivos planteados previamente, se procederá a tomar una muestra de cinco canteras de caliza ubicadas en el departamento del Cesar.

\section{Instrumentos}

Como esta investigación se lleva a cabo pormedio de un enfoque descriptivo, se maneja entonces para la obtención de los datos y la observación, para los que se emplea la lista de chequeo según Olivia (2009), en la que se concierta toda la información de las visitas realizadas a las respectivas canteras de extracción de la roca caliza en el departamento del Cesar, aplicándola en la variable minera. Posterior a esto se correlaciona con la información de la matriz de Leopold, realizada por Peláez (2017) para el parámetro ambiental, así como las normas Invías (2012) para determinar porcentajes del análisis químico para su uso en el mercado, de los resultados obtenidos por el PTO de cada contrato de concesión.

\section{Resultados}

Teniendo en cuenta la información obtenida a través de la Agencia Nacional de Minería (ANM), se establece que actualmente en el departamento del Cesar hay 35 canteras de caliza dedicadas a la extracción de la roca, de las cuales 17 se encuentran en etapa de exploración (ver figura1), en la que la mayoría de estas se encuentran en Valledupar y Bosconia; cinco en construcción y montaje, ubicadas en Valledupar, San Diego y La Paz (ver figura 2); y las doce restantes se encuentran en la etapa de explotación que corresponde a Valledupar, Bosconia, Curumaní y La Paz (ver figura 3).

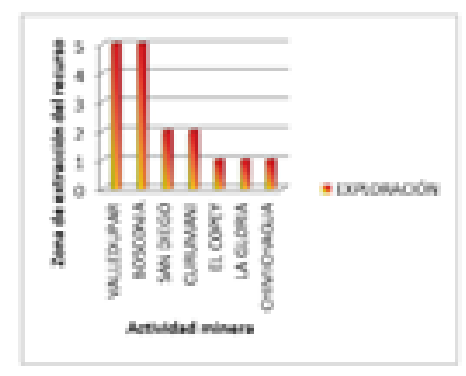

Figura 1. Etapa minera de exploración de las canteras de la caliza del departamento del Cesar Fuente: Islem Urbina (2015).

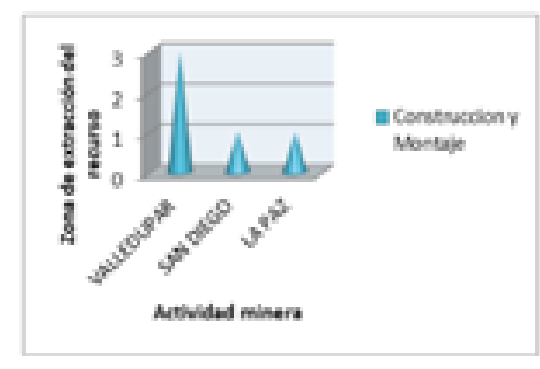

Figura 2. Etapa minera de construcción y montaje de las canteras de la caliza del departamento del Cesar. Fuente: Islem Urbina (2015). 


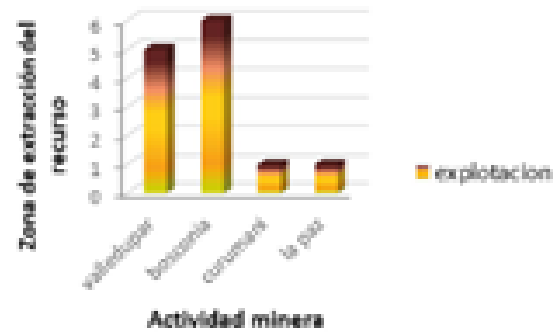

Figura 3. Etapa minera de explotación de las canteras de la caliza del departamento del Cesar

Fuente: Islem Urbina (2015).

\section{Diagnóstico geológico}

En este apartado se dividirán las cinco canteras estudiadas geológicamente, los estudios que se deben realizar de acuerdo con el PTO y los análisis realizados a la caliza por cada mina.

- Código_exp: 0184-20

Esta cantera se encuentra ubicada geológicamente en el grupo cogollo (ver figura 4); esta área está compuesta por calizas fosilíferas hacía la parte $\mathrm{NW}$, se observa caliza color gris claro con bivalvos, así como también calizas color gris oscuro con poca presencia de fósiles. La caliza encontrada en la superficie económicamente explotable es la mencionada primero, pero las que presentan escasos fósiles también son explotables.
Geográficamente se encuentran ubicadas en las siguientes coordenadas:

\begin{tabular}{|c|c|}
\hline $\mathrm{X}$ & $\mathrm{Y}$ \\
\hline 1598874 & 1022800 \\
\hline 1599900 & 1022800 \\
\hline 1599900 & 1023784 \\
\hline 1598874 & 1023784 \\
\hline
\end{tabular}

\section{Estudios geológicos}

Para este tipo de yacimiento, por lo general, no es necesaria la aplicación de los estudios geoquímicos y geofísicos, como es en este caso, de acuerdo con el PTO del contrato de concesión 018420 (2011), obtenido por la ANM, se pudo obtener la suficiente información a través de la interpretación superficial de la misma. Cabe recalcar que se aplica de igual manera para la realización de apiques o pozos.

Además de estos estudios, también se realzaron ensayos geológicos para determinar la calidad de la roca y la cantidad porcentual de los elementos químicos que conforman la caliza explotable, entre ellos se realizaron: ensayo de resistencia a la compresión inconfinada de cilindros, su objetivo fue caracterizar y clasificar un núcleo de roca intacta; determinación de la cohesión y el ángulo de fricción interna, 
utilizado para el cálculo de altura crítica del banco, obteniendo $\mathrm{c}=202,68$ y el ángulo de 32,40; resistencia al desgaste de los agregados (máquina de los ángeles), que fue del $20,9 \%$, teniendo en cuenta dicho porcentaje, se cataloga como muy resistente, los cuales pueden ser utilizados como base y subbase en construcciones, ya que, según las normas Invías (2012), debe ser máximo del $50 \%$.
Así mismo, dentro de los análisis químicos realizados a la caliza de explotación por la cantera se determinaron dos muestras el $42,5 \%$ de $\mathrm{CaO}$, el cual es bueno para el cemento, pero no es aprovechable para la industria del vidrio porque es menor al $50 \%$ del mismo compuesto químico.

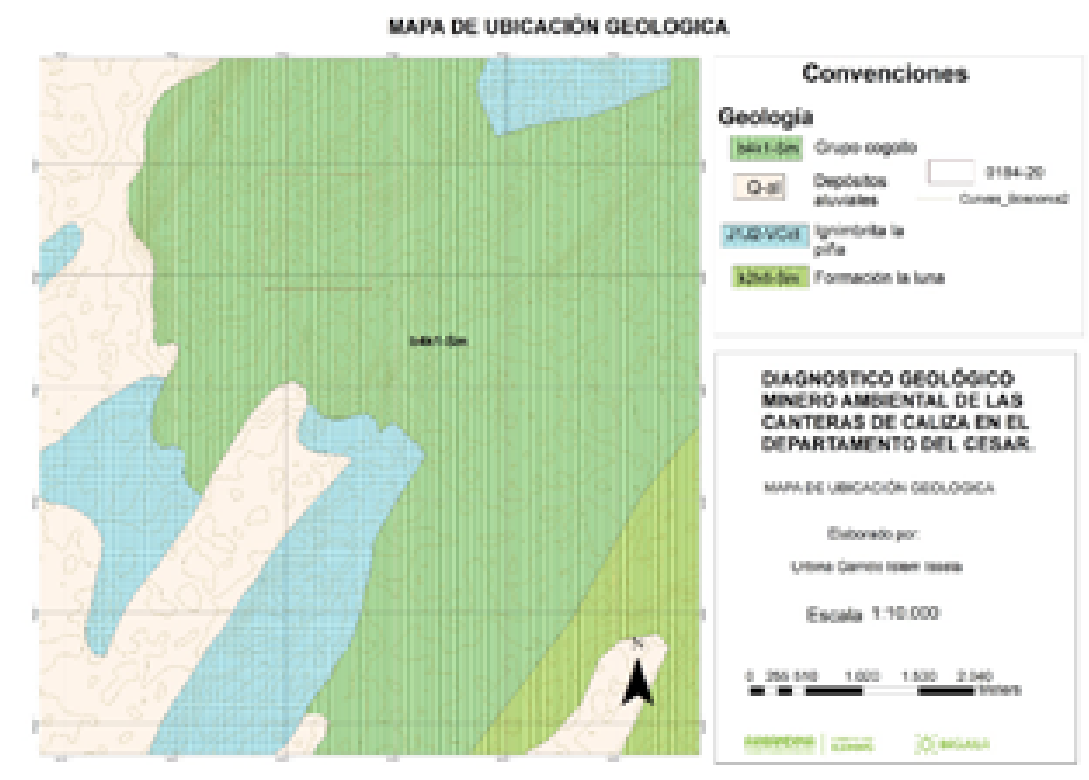

Figura 4. Ubicación geológica de la cantera de código 0184-20

Fuente: Islem Urbina (2015).

- Código_exp: 0190-20

Esta cantera también está ubicada geológicamente el grupo cogollo (ver figura 5), presentándose en esta área calizas fosilíferas de color gris claro, las que actualmente son las que están siendo explotadas, además, también se identifican calizas de gris oscuro con poca presencia de fósiles.

Geográficamente se encuentra ubicada en las siguientes coordenadas: 


\begin{tabular}{|c|c|}
\hline $\mathbf{X}$ & $\mathbf{Y}$ \\
\hline 1593750 & 1023500 \\
\hline 1594250 & 1023500 \\
\hline 1594250 & 1024000 \\
\hline 1593750 & 1024000 \\
\hline
\end{tabular}

\section{Estudios geológicos}

Ahora bien, según el PTO del número de solicitud 0190-20 (2009) obtenido por la ANM, se realizaron ensayos fisicomecánicos, en los que se obtuvo:

\begin{tabular}{|c|c|c|}
\hline $\begin{array}{c}\text { Resistencia } \\
\text { al desgaste }\end{array}$ & $\begin{array}{c}\text { Compresión } \\
\text { inconfinada }\end{array}$ & $\begin{array}{c}\text { Cohesión y } \\
\text { ángulo de } \\
\text { fricción }\end{array}$ \\
\hline $\begin{array}{c}19,1 \%- \\
18 \%\end{array}$ & $\begin{array}{c}42,06 \mathrm{~kg} / \\
\mathrm{cm}^{2}\end{array}$ & $\begin{array}{c}335,29 / \\
42,11\end{array}$ \\
\hline
\end{tabular}

Dentro del análisis químico, se manejan porcentajes similares de $\mathrm{SiO}$, $\mathrm{CaO}, \mathrm{Al} 2 \mathrm{O} 3, \mathrm{Fe} 2 \mathrm{O} 3, \mathrm{MgO}, \mathrm{TiO} 2$, $\mathrm{Na} 2 \mathrm{O}, \mathrm{K} 2 \mathrm{O}$, obteniendo de

\section{MAPA DE UBICACION GEOLOGICA}

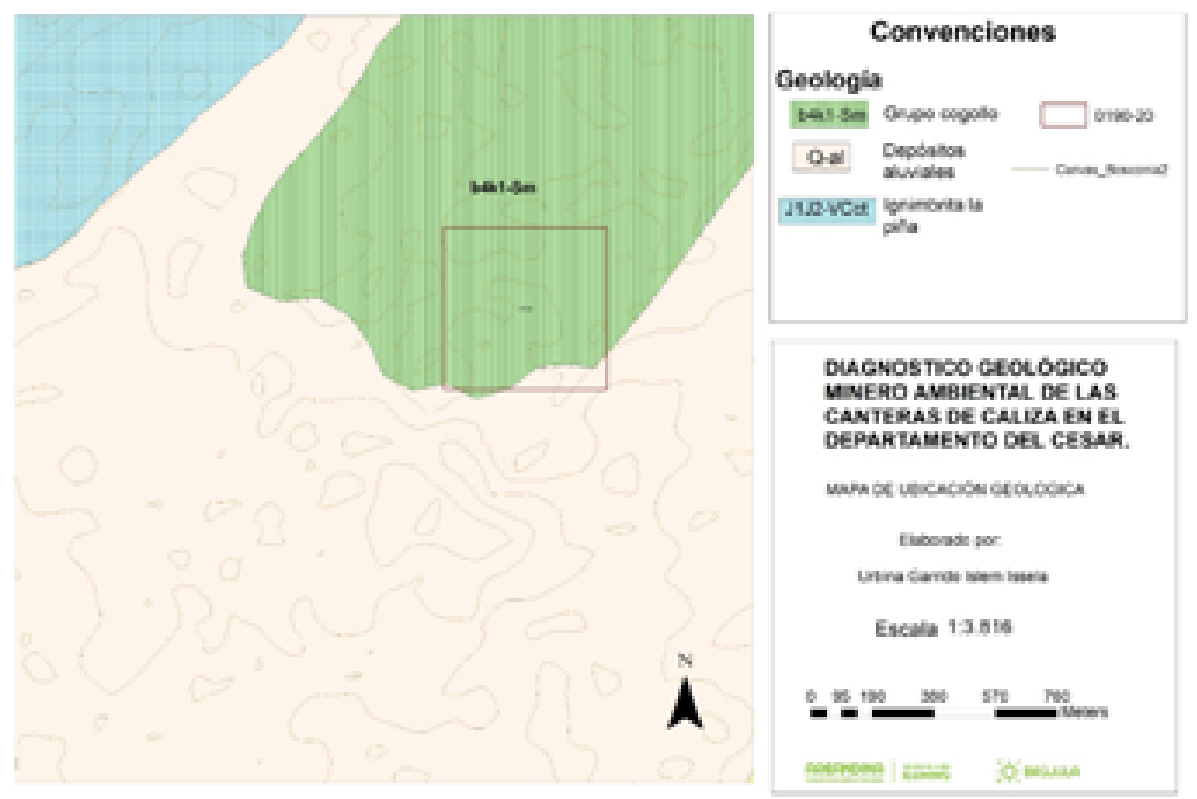

Figura 5. Ubicación geológica de la cantera de código 0190-20

Fuente: Islem Urbina (2015).

manera distintiva valores bajos en Al203 y Fe02 y alto en $\mathrm{Ca} 02$ y SiO2. 
De acuerdo con el porcentaje de desgaste se establece con una resistencia alta, así como también la cohesión alta genera o muestra una resistencia a la compresión. De la misma manera, los análisis químicos se ligan a la producción de construcción y cemento por ser valores similares entre $42-50 \%$ de $\mathrm{CaO} 2$ y $\mathrm{SiO} 2$.

\section{- Código_exp: 15056-1}

Esta cantera está ubicada geológicamente entre el grupo cogollo y la formación la luna (ver figura 6). No está demás mencionar que la más explotable es la caliza perteneciente a cogollo de la parte basal, conformado por capas de calizas grises de tonos claros a oscuros.
Geográficamente se encuentra ubicada en las siguientes coordenadas:

\begin{tabular}{|c|c|}
\hline$X$ & $Y$ \\
\hline 1598737 & 1027819,38 \\
\hline 1598737 & 1028719 \\
\hline 1599987 & 1028719 \\
\hline 1599987 & 1027819,38 \\
\hline
\end{tabular}

\section{Estudios geológicos}

No se realizan estudios geoquímicos ni geofísicos porque no es necesario, teniendo en cuenta la información obtenida de la geología superficial, que serían potencia, ubicación, forma y dirección.

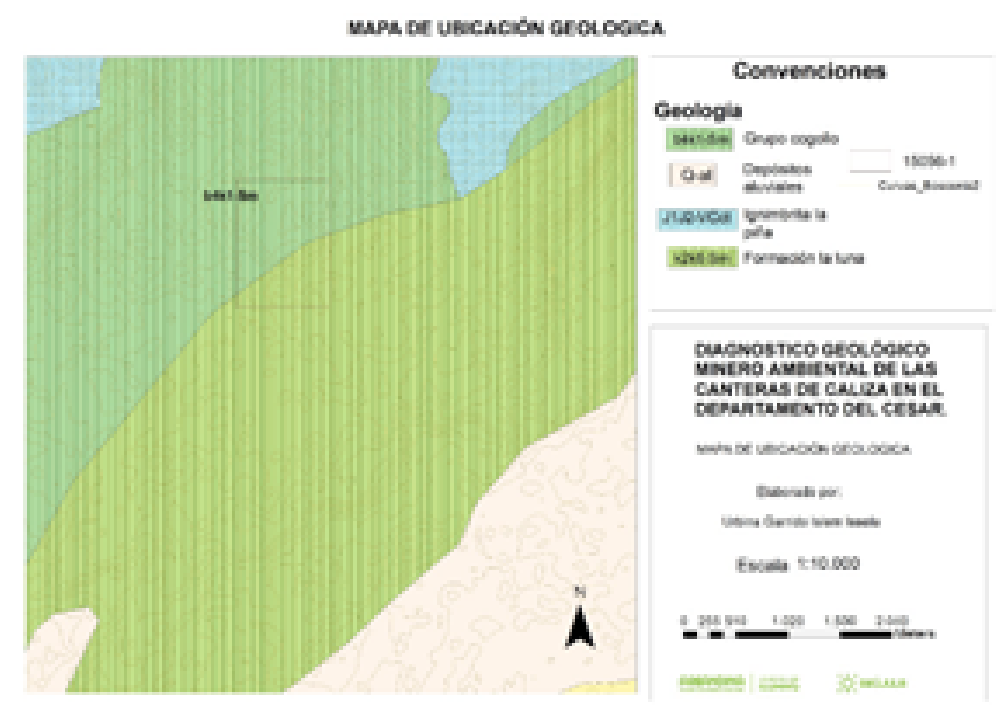

Figura 6. Ubicación geológica de la cantera de código 15056-1. Fuente: Islem Urbina (2015). 
Gracias a los destapes de afloramiento realizados en el área, la cantera, de acuerdo con esta información, tiene una idea clara del comportamiento del yacimiento en profundidad.

Basándose en el análisis químico realizado según el PTO del contrato de concesión 15056-1 (2009), obtenido por la ANM, se encuentra que las muestras oscilan entre $30,5 \%$ y $40,50 \%$ de $\mathrm{SiO} 2$, entre $6,21 \%$ y pérdida de calcinación de $\operatorname{los} 103$ a $1000{ }^{\circ} \mathrm{C}$ entre $36,33 \%$ y $39,19 \%$. Además, se realizó el ensayo de resistencia al desgaste arrojando un porcentaje del 23,4\%. De acuerdo con estos datos, según las normas Invías (2012), se puede incorporar al hormigón; cabe resaltar que la roca se establece como muy resistente.
- Código_exp: 0152-20

La cantera con el código 015220 está ubicada geológicamente en la formación la luna (ver figura 7). La escala es mucho más grande a comparación con la cartografía establecida en el área, presentándose, en realidad, en el grupo cogollo, conformado por capas de calizas grises de tonos claros a oscuros con intercalaciones irregulares de arcillolitas calcáreas.

Geográficamente se encuentra ubicada en las siguientes coordenadas:

\begin{tabular}{|c|c|}
\hline$X$ & $Y$ \\
\hline 1607000 & 1046000 \\
\hline 1607000 & 1047000 \\
\hline 1606500 & 1047000 \\
\hline 1606500 & 1046000 \\
\hline
\end{tabular}

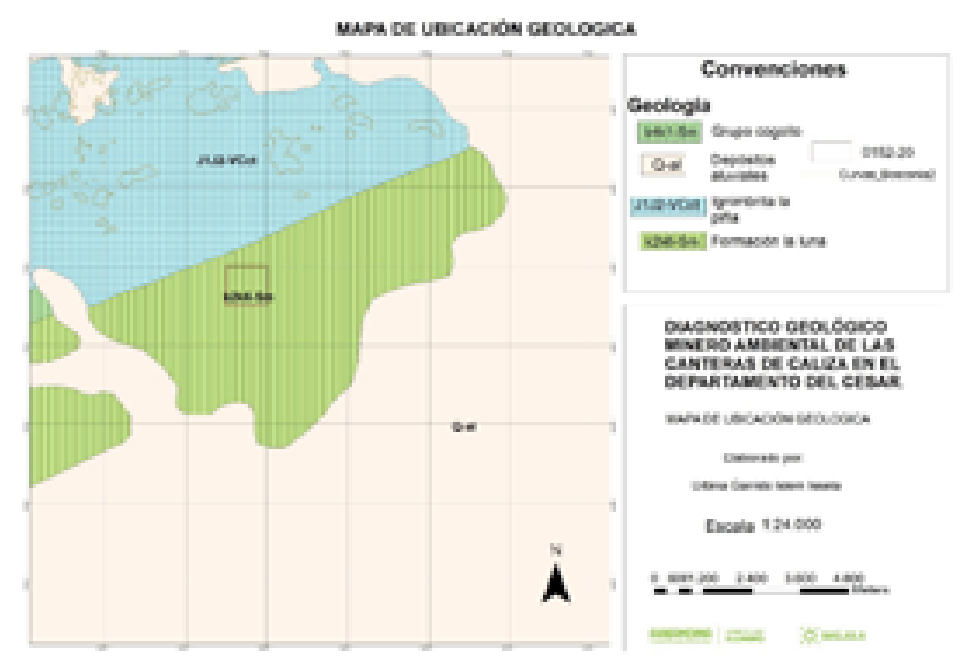

Figura 7. Ubicación geológica de la cantera de código 0152-20.

Fuente: Islem Urbina (2015). 


\section{Estudios geológicos}

Dentro de los análisis realizados según el PTO del número de solicitud 0152-20 (2007) obtenido por la ANM, el químico es de mayor interés, relacionado con valores de $\mathrm{CaO}$ del $56 \%$, lo que indica una caliza de alta pureza; los contenidos de sílice y alumina son muy bajos, en un promedio de $\mathrm{SiO} 2=1,3 \%$ y $\mathrm{A} 12 \mathrm{O} 3=0,4 \%$, lo que corresponde a calizas aptas para procesos químicos como fabricación de cal viva y usos industriales como el hierro y el acero.

- Código_exp: 0189-20

La cantera asignada con el código 0189-20 está ubicada geológicamente en la formación la luna, pero está a una escala mayor (ver figura 8); sin embargo, es el grupo cogollo cartográficamente a escala 1:10000, el cual está conformado por calizas grises de tonos claros a oscuros, el que se presenta en la cantera. De acuerdo con el PTO del número de solicitud 0189-20 (2007) obtenido por la ANM, al levantamiento geológico en los distintos frentes se identificaron tres mantos correspondiente a un yacimiento estratificado. Los niveles considerados como interés coinciden en su color gris claro con abundante presencia fosilífera y venas de calcita, baja densidad de fracturación y ausencia de alteraciones.

Geográficamente, se encuentra ubicada en las siguientes coordenadas:

\begin{tabular}{|c|c|}
\hline$X$ & $Y$ \\
\hline 1605000 & 1043000 \\
\hline 1606000 & 1043000 \\
\hline 1606000 & 1044000 \\
\hline 1605000 & 1044000 \\
\hline
\end{tabular}




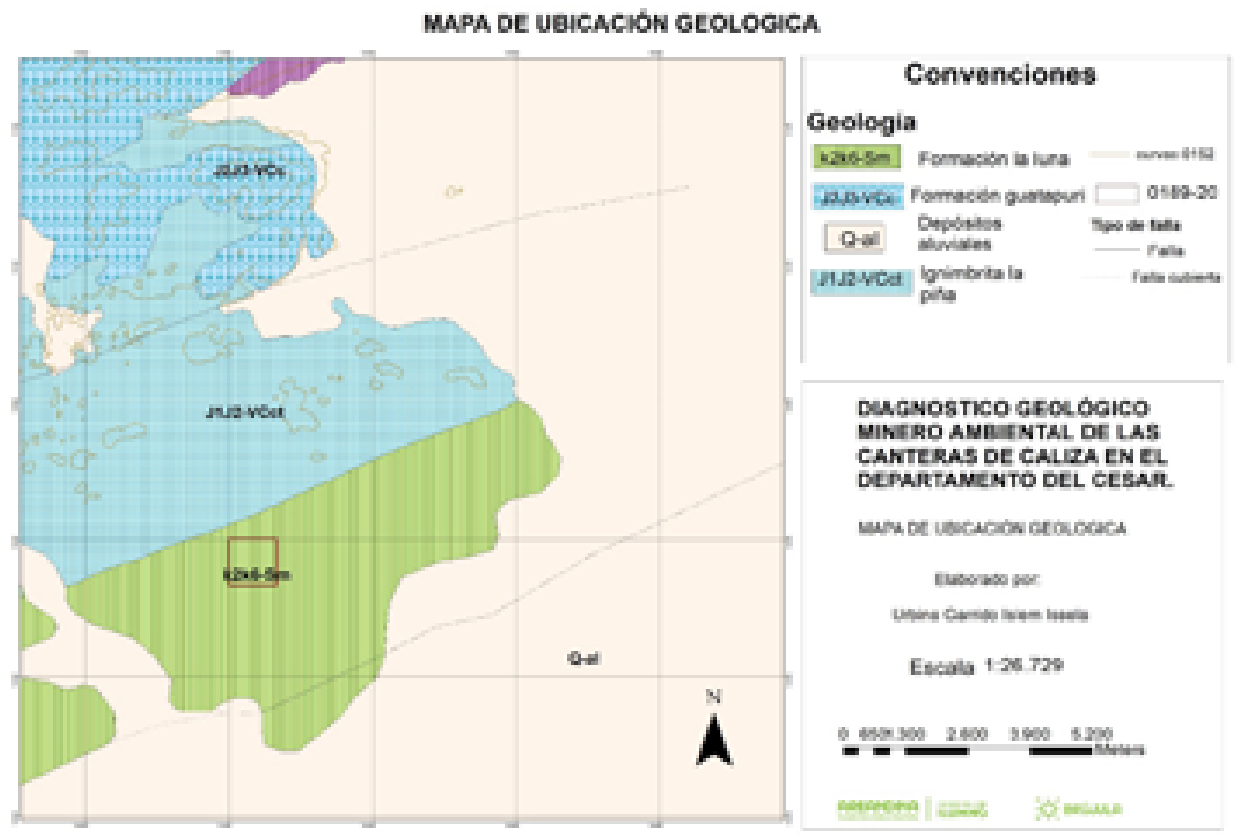

Figura 8. Ubicación

geológica de la cantera de código 0189-20

Fuente: Islem Urbina (2015).

\section{Estudios geológicos}

Dentro de los estudios mostrados en el PTO de la mina, los análisis químicos arrojaron un valor porcentual entre el $60 \%$ y $67 \%$, lo que indica que esta caliza es apta para la fabricación de cemento Portland, además de que el $\mathrm{CaO}$ es de $54,79 \%$, garantizando una pureza alta de la misma.

\section{Diagnóstico minero}

El método de explotación aplicado a las calizas es variado, la comparación de estos métodos se hace analizando las características del depósito de caliza como lo son: geometría, topografía, propiedades físicas y geomecánicas. Ahora bien, de acuerdo con Herbet (2006), el método más utilizado es el de banqueo, sus alternativas corresponden a banco único, banco escalonados ascendentes y bancos escalonados 
descendentes. Cada uno de ellos presenta ventajas y desventajas; por ejemplo, la primera tiene un buen rendimiento en cuanto a la perforación y voladura, pero su producción es baja, además de esto causa desviaciones en las perforaciones, esto a causa del espesor de los bancos o a la mala fracturación que ha tenido la roca; además, tiene un menor rendimiento en carga y transporte.

Con el banco escalonado descendente se tiene una ventaja desde el inicio que corresponde a la restauración, logrando además un mayor rendimiento en el arranque, cargue, transporte desde la explotación; también brinda mayor seguridad para el personal y maquinaria. Sin embargo, tiene como desventaja que es de largo plazo; también se exige construir la infraestructura completa para acceder a los niveles superiores desde el principio y a mayor distancia en el transporte, logrando una mayor inversión.

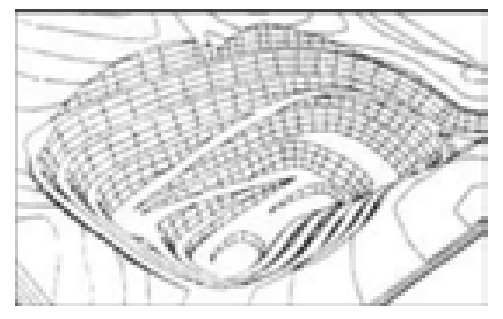

Figura 9. Esquema de un banco descendente Fuente: Herbet (2006).
Por último, se tiene el banco escalonado ascendente, el cual es uno de los métodos más utilizados por su facilidad de apertura de las canteras, además de la poca distancia de transporte hasta la planta de tratamiento, cabe recalcar que el frente siempre está activo. El inconveniente de este método se basa en la restauración, la cual se debe realizar finalizada el proceso de explotación. Del mismo modo, para la extracción de la caliza, son necesarios bancos de poca altura para así avalar la seguridad, además de esto, el establecimiento de maquinaria para construir el paso al frente y adecuación del área de interés. Por esta razón, el más adecuado para la extracción de la caliza es el de banco escalonado descendente por la seguridad tanto del trabajo como de los trabajadores.

\section{Diagnóstico ambiental}

Las repercusiones ambientales que está ocasionando la pequeña minería de caliza no están muy lejos de lo que ocasionan las de grande minería, la diferencia radica en que estas últimas emplean mayor mitigación en los impactos que generan. Unos de los más conocidos son las poluciones, estos 
generan un deterioro en el aire por el contenido de las partículas y gases emitidos a la hora de realizar la extracción. De la misma manera, la explotación de esta roca ayuda con la disminución en la calidad del suelo, producto de los procesos de meteorización, que aumentan su efectividad al quitar la capa vegetal que los cubren, facilitando su labor, ocasionando así la erosión de estos.

Del mismo modo, se tiene también el cambio geomorfológico, generado a partir de la extracción de la roca caliza en las canteras, las cuales no tienen en cuenta las afectaciones que generan a la variable paisajística; todo esto a razón de no tener un plan de tratamiento o restauración tanto del suelo como del paisaje.

Como es bien sabido, a la hora de fijar la explotación de algún mineral o material se debe tratar directamente con la naturaleza, es decir, el lugar del que procede lo que se quiere extraer, esto quiere indicar que se encuentran ligadas, por esto mismo, es necesario que se tenga en cuenta el medio en el que se trabaja y hacer lo posible para generar una minería bien hecha, en la que se trabajen con variables de máxima importancia, las cuales están preestablecidas como mitigación y prevención. Cabe recalcar que, además de lo realizado en esta investigación, se establece que dentro de las etapas mineras para la canteras la que más genera impacto ambiental corresponde a la etapa de construcción y montaje, esto basado en la matriz de Leopold (Peláez, 2017), la cual permite identificar diferentes ámbitos ambientales susceptibles a presentar cambios drásticos, pero que de igual manera se puedan dar de manera indirecta a las diversas zonas de estudio.

\section{Conclusión}

A lo largo de la trayectoria de la presente investigación se lograron determinar las diferentes etapas en las que se encontraban las canteras de caliza en el departamento del Cesar, las cuales han realizado sus respectivas extracciones aplicando conocimiento de estudios geológicos necesarios a realizar. Todas las canteras se abstuvieron de realizar estudios geoquímicos y geofísicos porque encontraban toda la información relevante en la geología superficial, cabe recalcar que la mayoría de la caliza explotada es utilizada para la elaboración de cemento y base de construcción, solo de la mina con código 0152-20 se puede 
extraer cal viva para procesos químicos, además de usos industriales en hierro y acero.

La formalización de la minería a pequeña escala requiere de mucha labor y dedicación, en la que una de las grandes dificultades es cumplir con los requisitos jurídicos establecidos por el PTO, además del ANLA; por esto mismo, se planteó el realizar una lista de chequeo para conocer en qué etapa se encontraban, si realizaban los estudios pertinentes y si se tenían en consideración las repercusiones ambientales, de las que se concluye que en realidad no las practican, más que todo por la inversión que tocaría implementar. No está de más mencionar que la idea no es eliminar la pequeña minería porque esto generaría mayor ilegalidad de las mismas, sino comenzar con aplicar metodologías que logren satisfacer las necesidades del titular de la cantera con respecto al cumplimiento de los requisitos mineros; por lo mismo, se establece aplicar el método de explotación para las canteras de caliza de banco escalonado descendente, ya que este brinda mayor seguridad para los operarios y maquinaria en toda la fase minera debido a la poca altura de los bancos. Cabe resaltar que el primer paso de este método es adecuar las vías en función de la facilidad de acceso a las áreas de interés, además de las áreas de cuidado y restauración de suelos que ya han sido descapotados.

Dentro de la variable ambiental, las zonas que más repercusiones ambientales han tenido son aquellas en las que se procede con la extracción de la roca, en la etapa de construcción y montaje, ya que es allí donde sucede la eliminación de la capa vegetal, aumentando la degradación física del medio, además del suelo y el aire, este por polución, por lo cual se establece la morfología como un cambio que tiene el área al momento de dar lugar a la explotación. Del mismo modo, también salen afectadas la flora y la fauna del área circundante, teniendo en cuenta la falta de mitigación de la contaminación empleada a la hora de la extracción.

Por último, la ciudad de Valledupar, ubicada en el departamento del Cesar, teniendo en cuenta las estadísticas planteadas anteriormente, esta categorizada como la mayor productora de la roca caliza, generando de la misma manera, un alto impacto ambiental donde residen cada una de estas canteras. 


\section{Referencias}

Aigneren, M. (2009). Diseños cuantitativos, análisis e interpretación de la información. Sociología en sus escenarios.

ANI (2012). Normas Invías. Sitio web: ftp:// ftp.ani.gov.co/Licitaci\%C3\%B3n\%20VJVGCLP\%20001-2016-M-1/Normas\%20 de $\% 20$ Ensayo $\% 20 \mathrm{de} \% 20$ materiales $\% 20$ para $\% 20$ carreteras/SECCI $\%$ C3\%93N\%20 600.pdf

Chaparro, E. (2000). La llamada pequeña minería: un renovado enfoque empresarial.

Chem, J. (1998). Factors Affecting the Rate of a Chemical Reaction. Journal of Chemical Education, 75(9).

Contento, J. (2014). Diseño del método de explotación del yacimiento de caliza módulo norte de la mina La Esperanza, municipio La Calera, Cundinamarca. https://repositorio.uptc.edu.co/bitstream/001/1525/1/TGT265.pdf.

CooperAcción, (2005). Formalización de la minería en pequeña escala en América Latina y el Caribe: un análisis de experiencias en el Perú.

Corponariño (s.f.). Diagnóstico minero ambiental distrito minero de La Llanada 2006-2007. Disponible en http://corponarino.gov.co/expedientes/publicaciones/diagnosticomineroambiental.pdf

CRC (2003). Diagnóstico geológico, mine- ro ambiental, social y económico distrito minero de fondas El Tambo-Cauca. Disponible en http://crc.gov.co/files/ConocimientoAmbiental $/$ mineria/MINERIA $\% 20$ TAMBO/Diagnostico\%20Distrito\%20Minero\%20de\%20Fondas\%20Tambo.pdf

D’Angelo, S. (2008). Población y muestra. 20/05/2015, de UNNE; UCP. Disponible en http://med.unne.edu.ar/sitio/multimedia/ imagenes/ckfinder/files/files/aps/POBLACI\%C3\%93N\%20Y\%20MUESTRA\%20 (Lic\%20DAngelo).pdf

González, L. (2013). Impacto de la minería de hecho en Colombia. Disponible en http:// www.uis.edu.co/webUIS/es/catedraLowMaus/lowMauss 13_1/terceraSesion/Impacto $\% 20 \mathrm{de} \% 201 \mathrm{a} \% 20$ mineria $\% 20 \mathrm{de} \% 20$ hecho $\% 20$ en $\% 20$ Colombia.pdf (consultado el 20 de mayo de 2015).

Herbet, J. (2006). Métodos de minería a cielo abierto. Disponible en http://oa.upm. es/10675/1/20111122_METODOS_MINERIA_A_CIELO_ABIERTO.pdf (consultado el 20 de mayo de 2015).

MMSD. (2012). Final report. Breaking New Ground: Mining, Minerals and Sustainable Development.

Olivia, P. (2009). Listas de chequeo como técnicas de control. Disponible en http:// www.minsal.gob.cl/portal/url/item/7cf9e499a55c4cc7e04001011f016c69.pdf

Recasens, A. (2005). Fundamentos epistemológicos, metodológicos y teóricos que sustentan un modelo de investigación cualitativa en las ciencias sociales. 